\title{
Dimple Colloids with Tunable Cavity Size and Surface Functionalities
}

\author{
Yong Guo, ${ }^{\circledR}$ Bas G. P. van Ravensteijn, ${ }^{\dagger}$ and Willem K. Kegel* \\ Van't Hoff Laboratory for Physical and Colloid Chemistry, Debye Research Institute, Utrecht University, Padualaan 8, $3584 \mathrm{CH}$ \\ Utrecht, The Netherlands
}

\section{Supporting Information}

\begin{abstract}
Dimple colloids with well-defined cavities were synthesized by a modified dispersion polymerization. The key step in the procedure is the delayed addition of crosslinkers into the reaction mixture. By systematically studying the effect of the delayed addition time and the concentration of the cross-linker on the resulting particle morphology, we identified the dominating driving force that underlies dimple formation. The delayed addition of cross-linkers results in colloids with a core-shell morphology consisting of a core rich in linear polymers and a cross-linked shell. This morphology was confirmed by selectively etching non-cross-linked material using dimethylformamide. With polymerization proceeding, consumption of monomers present in the swollen particles leads to contraction of the particles, which is larger for the core composed of linear polymers compared to the stiffer cross-linked shell. To accommodate this decrease in volume, the outer cross-linked shell has to buckle, resulting in a well-defined dimple. Furthermore, we extended the procedure to incorporate functional monomers, yielding chemically modifiable dimple particles. Subsequently, we showed that by leveraging the core-shell structure, these dimple particles can be used to prepare dumbbell-shaped colloids with one hollow and one solid lobe. These partially hollow anisotropic particles assemble into strings with well-defined orientations in an alternating current electric field.
\end{abstract}

\section{INTRODUCTION}

Colloidal particles with an anisotropic shape and/or composition receive increasing attention due to their potential use as building blocks for the fabrication of (photonic) colloidal assemblies, ${ }^{1-3}$ as model systems for atoms or molecules, ${ }^{4}$ as drug delivery vehicles, ${ }^{5}$ and as self-propelling objects. ${ }^{6,7}$ Sparked by these possible avenues for applications, colloidal synthetic techniques that give access to, for example, dumbbell-shaped, ${ }^{8,9}$ Mickey Mouse, ${ }^{10}$ cubic, ${ }^{11}$ and ellipsoidal ${ }^{12}$ particles were developed. Another interesting class of anisotropic colloids is so-called dimple particles, whose surfaces feature one or multiple cavities. This particular particle morphology renders these particles ideal candidates for synthetic analogues of red blood cells to study their permeation behavior and to use them as primary constituents of depletion-driven lock-and-key assemblies. ${ }^{13-16}$ The preferred route to make these colloids in high yield relies on the delayed addition of a cross-linker into emulsion or dispersion polymerization systems. ${ }^{17-19}$ Despite the versatility and simplicity of the method, two conflicting mechanisms underlying cavity formation were reported in the current literature. ${ }^{17-21}$ Huang et al., ${ }^{17}$ Cheng et. al,${ }^{18}$ and Xie et. $\mathrm{al}^{20}$ proposed a dimple formation mechanism based on the inhomogeneous distribution of cross-linkers and subsequent phase separation, while Peng et. $\mathrm{al}^{19}$ and Meester et. $\mathrm{al}^{21}$ suggested that buckling is the main driving force of the dimple formation.
Here, we present a systematic study on the effect of the delayed addition time and the concentration of cross-linkers on the resulting particle morphology to elucidate the dominating driving force responsible for dimple formation. We observed that the delayed addition of cross-linkers generates colloids with a distinct core-shell structure in which the core, rich in linear polymers, is encapsulated by a cross-linked shell of uniform thickness. With the polymerization proceeding, the monomer-swollen core undergoes a volumetric contraction that induces the elastic cross-linked shell to buckle, yielding a well-defined cavity. In agreement with the buckling mechanism, the tendency toward buckling and therefore the cavity dimensions are correlated to $r / s$, that is, the ratio of the particle radius $r$ over the shell thickness $s$. Experimentally, $r / s$ is readily tunable by controlling the delayed addition time and the concentration of the cross-linker. Furthermore, the approach was easily extended to include functional monomers for the preparation of chemically modifiable dimple colloids, expanding the application scope of these colloids. Lastly, the unique core-shell structure of these seed particles allows for the preparation of (chemically) anisotropic dumbbell-shaped colloids with one hollow and one solid lobe. To exemplify the use of these particles, their anisotropic density was used to

Received: February 27, 2019

Revised: April 20, 2019

Published: May 17, 2019 
guide the directional alignment of these new model colloids in an externally applied alternating current (AC) electric field.

\section{EXPERIMENTAL SECTION}

2.1. Materials. Styrene (St, 99\%), divinylbenzene (DVB, 55\% mixture of isomers, tech. grade), 4-vinylbenzyl chloride (VBC, $\geq 90 \%$, tech. grade), $\mathrm{N}$-isopropylacrylamide (NIPAM, 97\%), fluoresceinamine (mixture of isomers), dimethylformamide (DMF, $\geq 99 \%$ ), poly(vinylpyrrolidone) (PVP, K30, $M_{\mathrm{w}}=40 \mathrm{~kg} / \mathrm{mol}$ ), and poly(vinyl alcohol) (PVA, $M_{\mathrm{w}}=85-124 \mathrm{~kg} / \mathrm{mol}, 87-89 \%$ hydrolyzed) were obtained from Sigma-Aldrich. Azobis(isobutyronitrile) (AIBN, 98\%) was purchased from Acros Organics. Glycidyl methacrylate (GMA, $\geq 97.0 \%$ ) was purchased from Fluka Analytical. Sodium dodecyl sulfate (SDS) was purchased from BDH. Hydroquinone (99\%) was obtained from Riedel-de Haën. Ethanol (p.a., ACS reagent) was purchased from Merck. 2,2'-Azobis(2,4-dimethylvaleronitrile) (V65) was obtained from Wako chemicals $\mathrm{GmbH}$. All chemicals were used as received. The water used throughout all of the experiments was purified using a Milli- $Q$ water purification system.

2.2. Synthesis of Polystyrene Dimple Particles. Typically, PVP (5 g, $0.125 \mathrm{mmol})$, ethanol $(130 \mathrm{~mL})$, AIBN (0.136 g, 0.8 $\mathrm{mmol})$, and water $(14 \mathrm{~mL})$ were charged into a $250 \mathrm{~mL}$ three-neck round-bottom flask. Dissolution of PVP and AIBN was aided by sonicating the mixture. Subsequently, St $(10 \mathrm{~mL}, 87 \mathrm{mmol})$ was added. The obtained solution was constantly stirred with a magnetic stirrer under nitrogen flow. After $30 \mathrm{~min}$, the flask was immersed in a $70{ }^{\circ} \mathrm{C}$ water bath to initiate polymerization. After $1.5 \mathrm{~h}$ of polymerization, a premixed solution containing DVB $(0.3 \mathrm{~mL}, 2$ mmol), St $(2.5 \mathrm{~mL}, 22 \mathrm{mmol})$, and ethanol $(5 \mathrm{~mL})$ was added dropwise over a period of approximately $30 \mathrm{~min}$. The reaction was allowed to proceed for an additional $22.5 \mathrm{~h}$. The obtained colloidal particles were washed using centrifugation and redispersion cycles with ethanol $(2 x)$ and water $(2 x)$, respectively. Lastly, the particles were stored in water. To monitor the particle formation with time, samples $(300 \mu \mathrm{L})$ were withdrawn from the reaction mixture 0.1, $0.25,0.5,1,2,4,8,10,23$, and $24 \mathrm{~h}$ after the start of the polymerization. The sample $(150 \mu \mathrm{L})$ was used to gravimetrically measure the solid content and monomer conversion, while the remainder of the aliquot was used to determine particle size and morphology using transmission electron microscopy (TEM). Before TEM analysis, the samples were washed with ethanol $(2 \times)$ and water $(2 \times)$, respectively.

2.3. DMF-Mediated Etching of Dimple Colloids: Proving Core-Shell Morphology. Typically, a dimple particle dispersion as synthesized following the procedure described in Section $2.2(15 \mu \mathrm{L}$, solid content $=18 \%)$ was centrifuged, washed with DMF $(3 \times)$, and subsequently redispersed in DMF $(1 \mathrm{~mL})$. The dispersion was stirred for $24 \mathrm{~h}$ at $80{ }^{\circ} \mathrm{C}$ to selectively solubilize non-cross-linked polystyrene. ${ }^{22}$ After the etching procedure, the sample was centrifuged and washed with DMF $(3 \times)$ and water $(3 x)$. After the washing procedure, the particles were stored in water.

2.4. Synthesis of Functional Dimple Polystyrene Colloids. The synthetic procedure is analogous to the one described in Section 2.2. In addition to St $(1.5 \mathrm{~mL}, 14 \mathrm{mmol})$, DVB $(0.36 \mathrm{~mL}, 2.7 \mathrm{mmol})$, and ethanol $(5 \mathrm{~mL})$, the solution, which was added after formation of the initial non-cross-linked polystyrene nuclei/particles, also contained either VBC $(1.5 \mathrm{~mL}, 11 \mathrm{mmol})$, GMA $(1.5 \mathrm{~mL}, 11 \mathrm{mmol})$, or $\operatorname{NIPAM}(1.2 \mathrm{~g}, 11 \mathrm{mmol})$ as functional comonomers. This solution was added dropwise to the initial reaction mixture $1.5 \mathrm{~h}$ after the start of the polymerization. The reaction was allowed to proceed for an additional $22.5 \mathrm{~h}$. The final products were washed with ethanol $(2 \times)$ and water $(2 \times)$. Lastly, the particles were stored in water.

2.5. Covalent Attachment of Fluoresceinamine to Chlorinated Dimple and Dumbbell-Shaped Particles. A dispersion containing chlorinated dimple or dumbbell-shaped particles $(55 \mu \mathrm{L}$, solid content $=18 \%)$ was centrifuged and washed with DMF $(3 \times)$ (see Section 2.6 for synthesis of dumbbells-shaped colloids). After the washing steps, the colloids were redispersed in DMF $(1 \mathrm{~mL})$. Subsequently, fluoresceinamine $(3.5 \mathrm{mg}, 0.01 \mathrm{mmol})$ was added, and the dispersion was heated at $90{ }^{\circ} \mathrm{C}$ for $24 \mathrm{~h}$. During the coupling of the dye, the reaction vial was covered with aluminum foil to avoid bleaching of the dye. The particles were sequentially washed with DMF and ethanol to remove excess dye. The labeled particles were dispersed in water and stored in the dark.

2.6. Preparation of the Partially Hollow Dumbbell-Shaped Particles Suitable for Directed Assembly in an AC Electric Field. The seed particles for the synthesis of the partially hollow dumbbell-shaped particles were prepared by using the method in Section 2.2, except that no water was added in the initial mixture and the delayed addition time of the cross-linker was $4 \mathrm{~h}$ after the start of the polymerization. By doing so, larger dimple particles with thinner shells were obtained. For the preparation of dumbbell-shaped particles, V65 (15 mg, $0.06 \mathrm{mmol})$, DVB (11 $\mu \mathrm{L}, 0.08 \mathrm{mmol})$, St $(750 \mu \mathrm{L}, 6.6 \mathrm{mmol})$, and $1 \mathrm{wt} \%$ aqueous PVA solution $(9 \mathrm{~mL})$ were mixed in an elongated $25 \mathrm{~mL}$ vial and emulsified at $8000 \mathrm{rpm}$ for 6 min by using an IKA T-25 Ultra Turrax with an S25N 10G dispersing tool. Subsequently, the seed dispersion $(2.5 \mathrm{~mL}$, solid content $=$ $3.5 \%)$ was added, and the vial was placed on a roller table for $24 \mathrm{~h}$. After that, the vial was immersed into an oil bath at $70{ }^{\circ} \mathrm{C}$ to polymerize the added monomers for $24 \mathrm{~h}$. The product was washed with ethanol $(2 x)$ and water $(2 x)$ and stored in water. The obtained colloids were then converted to the targeted dumbbells with one solid and one hollow lobe by following the DMF-mediated etching procedure as described in Section 2.3.

2.7. Assembly of Partially Hollow Dumbbell-Shaped Particles in an AC Electric Field. Dumbbell-shaped particles with one solid and one hollow lobe were dispersed in DMF (solid content $\approx 0.2 \%$ ). For the alignment of the colloids in an AC electric field, a custom-made capillary was fabricated as follows: Two copper wires (diameter $=0.05 \mathrm{~mm})$ were passed through a capillary $(4.5 \mathrm{~cm} \times 1$ $\mathrm{mm} \times 0.1 \mathrm{~mm}$ ) and closely put to the walls of the capillary separately. The particle dispersion was filled into this capillary after which the two ends of the capillary were sealed using two-component epoxy glue. The capillary was placed on a microscope slide (Menzel-Gläser) and fixed using tape. Subsequently, the capillary with particle dispersion was exposed to an AC electric field $(40 \mathrm{~V} / \mathrm{mm}$, frequency of $1 \mathrm{MHz}$ ). After several minutes, nearly all of the particles were assembled into chains in the field direction. The results were directly observed and recorded by optical microscopy.

2.8. Characterization. Transmission electron microscopy pictures were taken with a Philips Tecnai 10 electron microscope typically operating at $100 \mathrm{kV}$. The samples were prepared by drying a drop of diluted aqueous dispersion on top of polymer-coated copper grids. In order to freeze-dry TEM samples, $1 \mu \mathrm{L}$ of dispersion was placed on top of a polymer-coated copper grid. The grid was vitrified in liquid nitrogen and mounted onto a cryo-transfer unit, which was brought to vacuum $\left(10^{-4} \mathrm{~Pa}\right)$. The temperature was increased to -90 ${ }^{\circ} \mathrm{C}$ at $5{ }^{\circ} \mathrm{C} / \mathrm{min}$ and kept constant for roughly $6 \mathrm{~h}$ under vacuum to allow the water to sublime. The shell thickness and cavity size were determined by using iTEM software.

Scanning electron microscopy images were taken with a Philips SEM XL FEG 30 typically operating at $5-10 \mathrm{kV}$. The dried samples of particles were sputter-coated with platinum (thickness $=6 \mathrm{~nm}$ ) prior to imaging.

Infrared (IR) spectra were obtained using a PerkinElmer Frontier FT-IR/FIR spectrometer. The attenuated total reflectance (ATR) mode was used. Measurements were carried out on powders obtained by drying the particle dispersions.

Confocal microscopy pictures were recorded with a Nikon TE $2000 \mathrm{U}$ laser scanning confocal microscope equipped with a Nikon C1 scanning head in combination with an Ar-ion laser (488 nm, Spectra Physics), a HeNe laser (543.5 nm, Melles Griot), and an oil immersion lens (100× Nikon Plan Apc, NA 1.4).

Optical microscopy (OM) images were taken with a Nikon Ti-E inverted microscope. The microscope was equipped with a Nikon TIRF NA $1.49100 \times$ oil immersion objective, intermediate magnification of $1.5 \times$, and a Hamamatsu ORCA Flash camera. 


\section{RESULT AND DISCUSSION}

3.1. Influence of the Cross-Linker Addition Time after Initiation of Polymerization. As mentioned in the

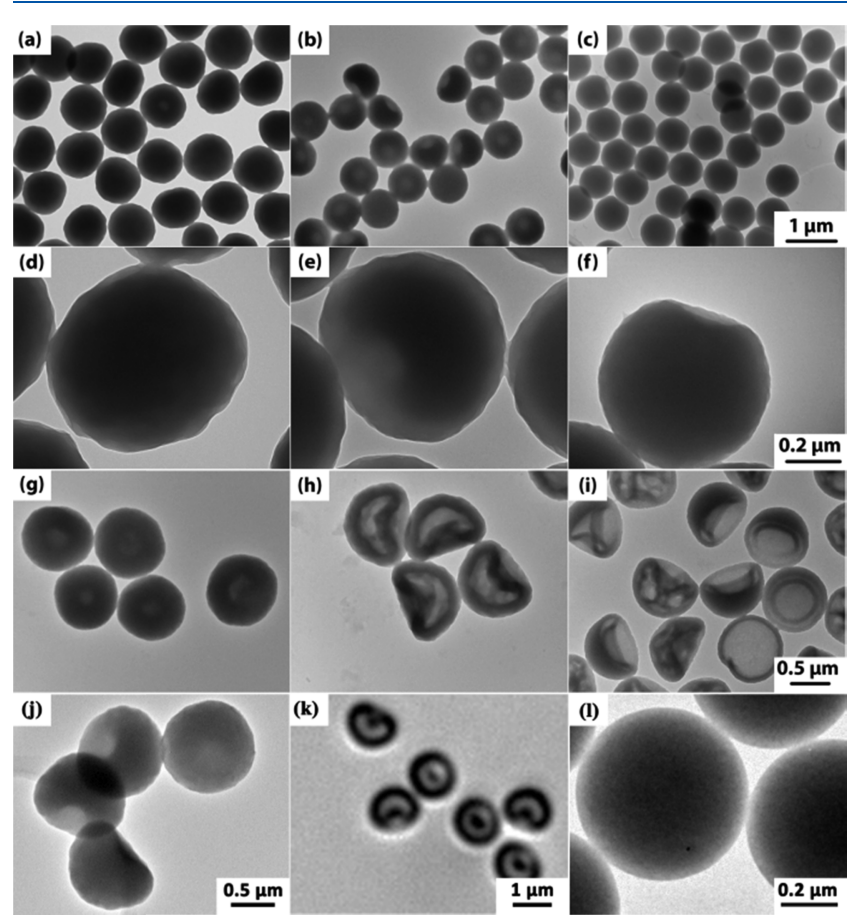

Figure 1. TEM images of polystyrene particles obtained by varying the delayed time after which the cross-linker (divinylbenzene, DVB) was added to the reaction mixture: (a) 0.1, (b) 1.5, and (c) 6 h. (d-f) surface morphology of the particles shown in panels (a), (b), and (c), respectively. (g-i) colloids shown in panels (a), (b), and (c), respectively, after etching with DMF. (j) TEM image of freeze-dried PS-1.5h. (k) Optical microscopy image of PS-1.5h dispersed in water. (l) TEM image of PS- $18 \mathrm{~h}$.

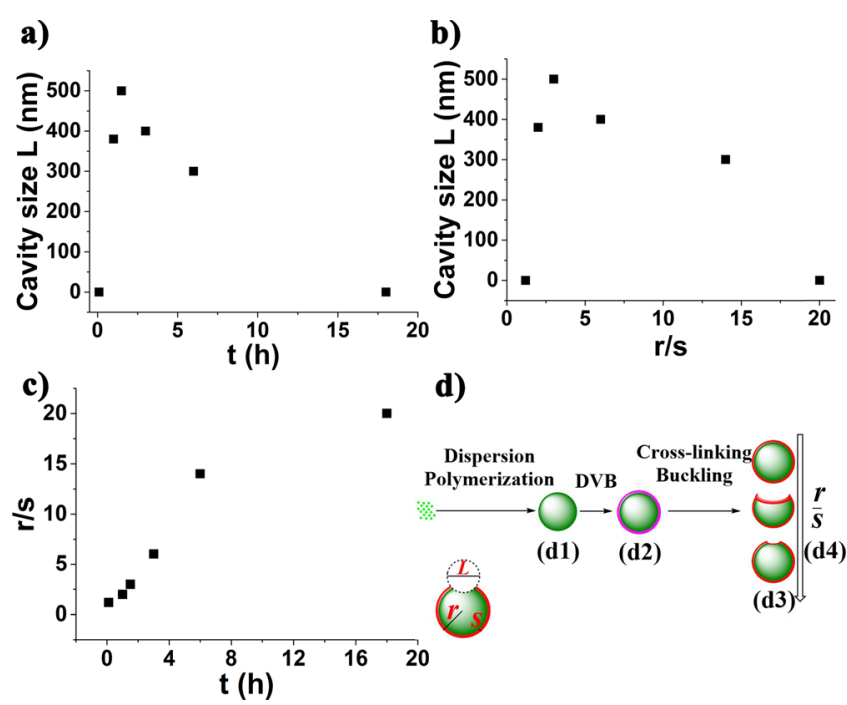

Figure 2. Cavity diameter $L$ as a function of (a) addition time of cross-linkers and (b) the particle radius $r$ over the shell thickness $s(r)$ s). (c) $r / s$ as a function of addition time. (d) Schematic illustration of the preparation of dimple particles. (d1) St-swollen PS particle. (d2) St-swollen PS core and (St/DVB)-swollen PS shell. (d3) Linear PS core and cross-linked $\mathrm{P}(\mathrm{St}-\mathrm{co}-\mathrm{DVB})$ shell. (d4) Cavity formation is determined by $r / s$.

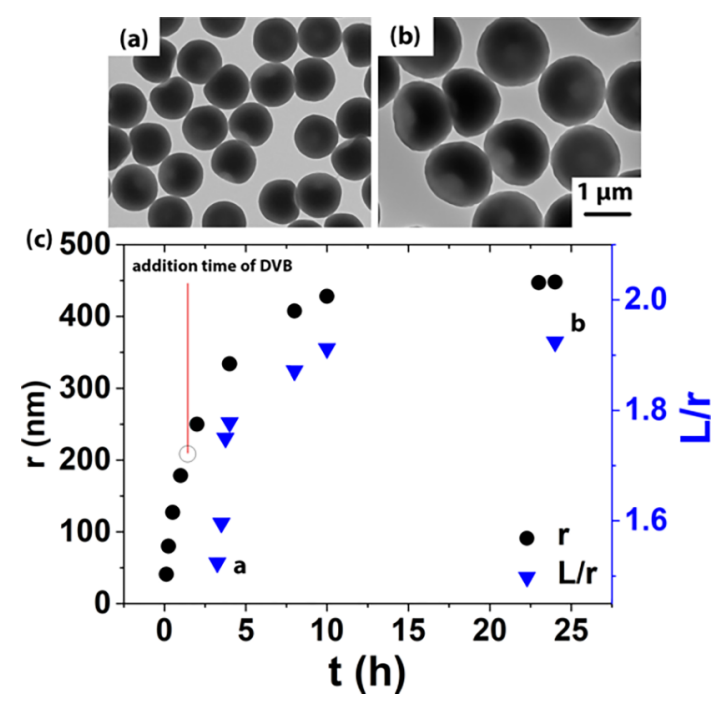

Figure 3. TEM images of PS-1.5h at (a) 3.25 and (b) $24 \mathrm{~h}$ from the start of the dispersion polymerization. (c) Time evolution of the particle radius ( $r$, filled circle, black) of PS-1.5h and ratio of the cavity diameter over the particle radius $(L / r$, filled triangle, blue) of PS-1.5h.
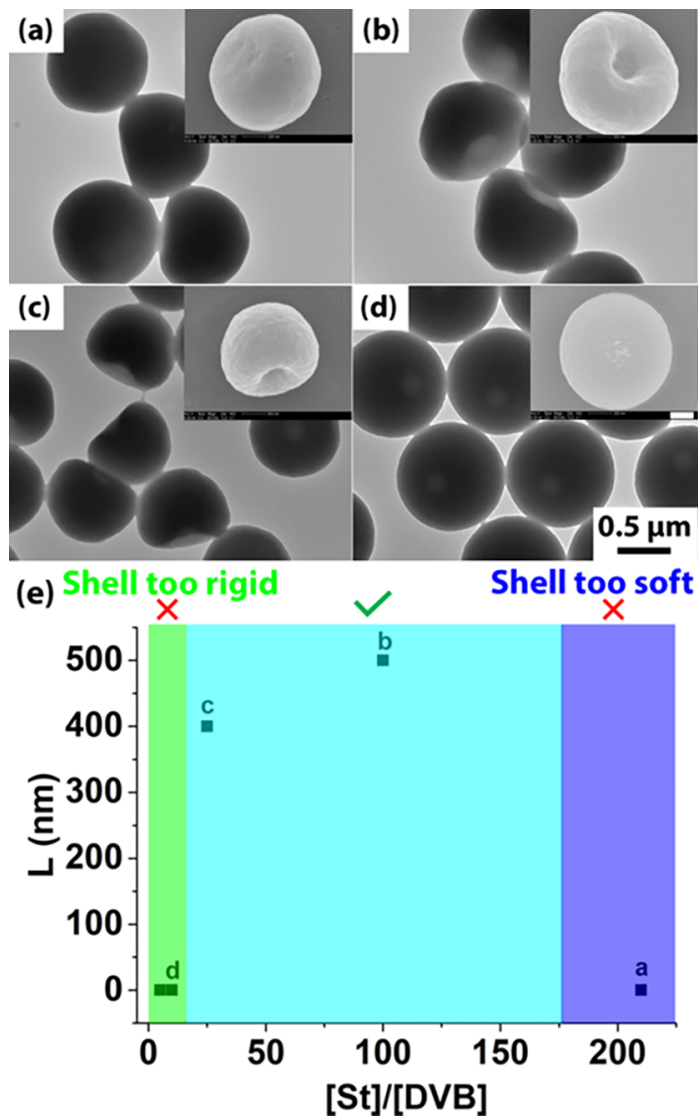

Figure 4. TEM images of particles synthesized using a molar ratio of total styrene to DVB of (a) 210:1, (b) 100:1, (c) 25:1, and (d) 10:1, respectively. (e) Cavity diameter $L$ as a function of total molar ratio of St to DVB. The addition time of the cross-linker was fixed at $1.5 \mathrm{~h}$ after the start of the initial polymerization. Scale bars of SEM images: $200 \mathrm{~nm}$.

Introduction, the delayed addition of cross-linkers to conventional emulsion or dispersion polymerizations is a versatile synthetic strategy toward particles with controlled surface 


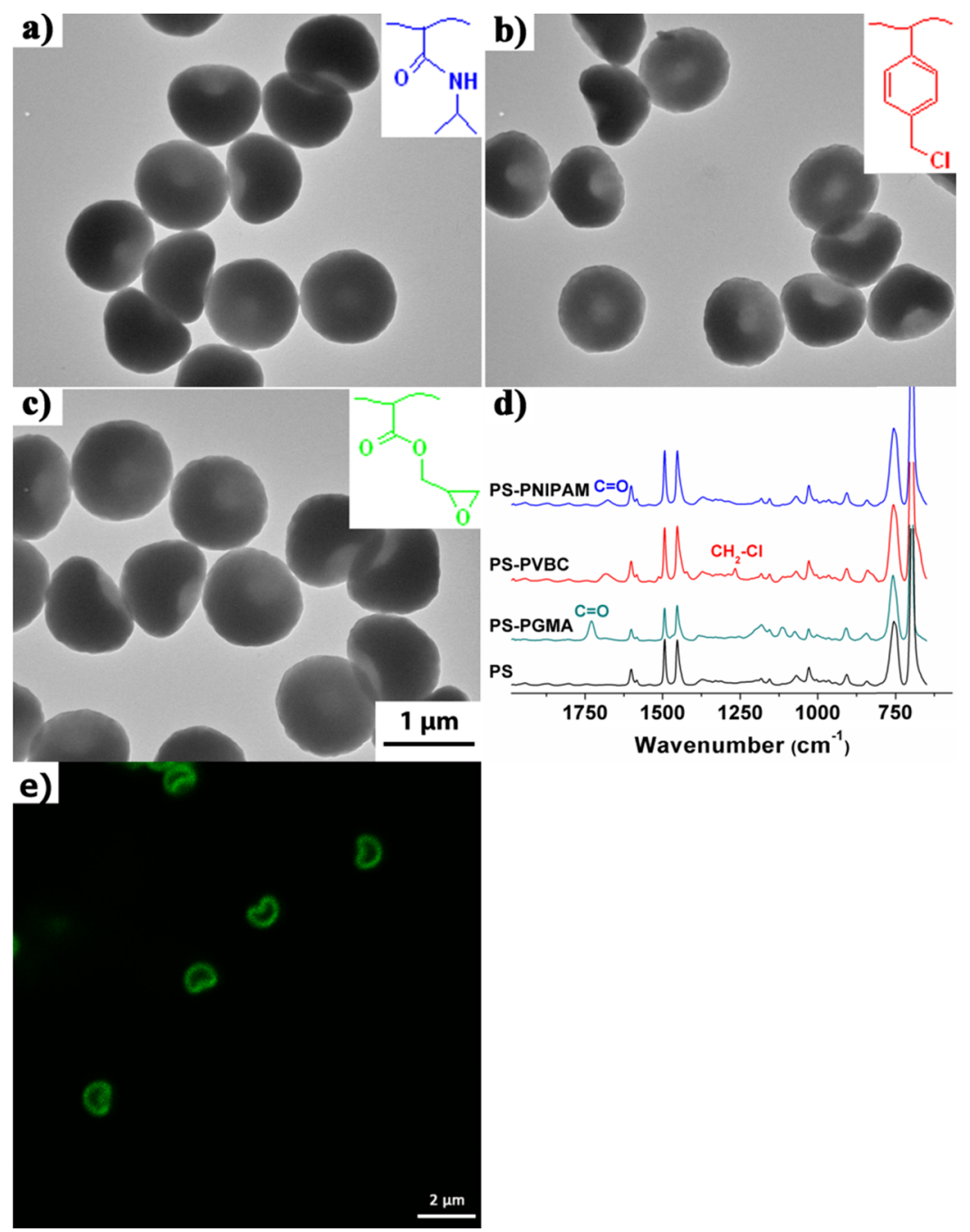

Figure 5. TEM images of surface-functionalized dimple particles (a) PS-PNIPAM, (b) PS-PVBC, and (c) PS-PGMA. (d) FTIR spectra of PSPNIPAM, PS-PVBC, PS-PGMA, and non-functionalized dimple PS particles (from top to bottom). Because the spectra are dominated by the large PS bulk of the colloids, the intensities of the signals corresponding to the functional monomers are relatively low. (e) Confocal microscopy image of PS-PVBC after surface modification with fluoresceinamine.

morphology, including (multi)dimple particles. ${ }^{17-19}$ To investigate what driving force governs dimple formation, we first selected suitable addition times of cross-linker (DVB), by monitoring the time evolution of the particle size and monomer conversion of a dispersion polymerization of solely styrene (see Supporting Information S1 for the details). Based on the kinetics of this polymerization, DVB addition times $(0.1,1,1.5,3,6$, and $18 \mathrm{~h}$ after the start of the initial polymerization) spanning the identified time window in which particles form and grow were selected.

Addition of DVB after $0.1 \mathrm{~h}$ (PS-0.1h) resulted in slightly aspherical microspheres with a rough surface, and no pronounced cavity was observed (Figures 1a,d and 2). Addition of DVB after $18 \mathrm{~h}$ (PS-18h) led to monodisperse microspheres with a smooth surface, and also no pronounced cavity was observed (Figures 11 and 2). However, when DVB was added after $0.1-18 \mathrm{~h}$, dimple particles with different cavity sizes were observed. For example, when DVB was added after $1.5 \mathrm{~h}$ (PS-1.5h) and $6 \mathrm{~h}$ (PS-6h), the low-contrast region in the TEM images in combination with their characteristic bowlshaped geometry indicates the presence of a cavity with an average diameter of approximately $500 \mathrm{~nm}$ (Figure 1b,e, PS- 1.5h) and $300 \mathrm{~nm}$ (Figure 1c ,f, PS-6h). Imaging PS-1.5h after being freeze-dried with TEM (Figure 1j) and dispersed in water with optical microscopy (Figure $1 \mathrm{k}$ ) confirmed that the dimpled particle shape is the true morphology of the colloids and the observed anisotropy cannot be attributed to artifacts due to sample drying as required for electron microscopy analysis. Particles with the largest obtainable cavity size were obtained when DVB was added after $1.5 \mathrm{~h}$ of polymerization (Figure 2). From this set of experiments, it was concluded that the cavity dimension sensitively depends on the DVB addition time.

3.2. Formation Mechanism of Dimple Particle. Two conflicting mechanisms underlying cavity formation were reported in the current literature: one is based on the inhomogeneous distribution of cross-linkers and subsequent phase separation between cross-linked domains and unreacted monomers, while the other relies on buckling of an outer elastic shell. To differentiate between the two competing mechanisms, the distribution of cross-linked domains in the colloids was investigated. If the inhomogeneous distribution of cross-linkers and subsequent phase separation are responsible, the cross-linked areas should be located around the 
(a)
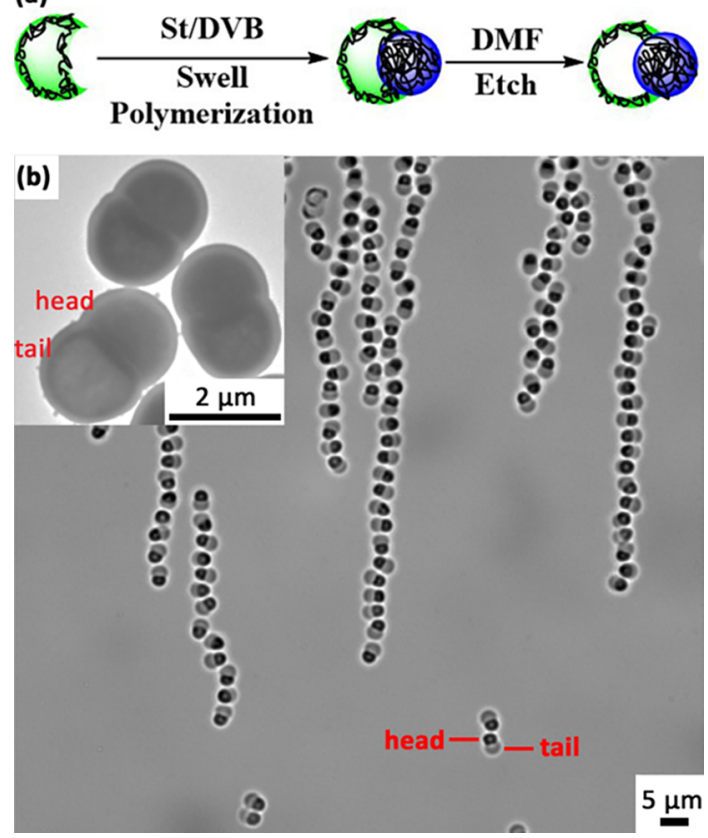

Figure 6. (a) Schematic representation of the preparation of partially hollow dumbbell-shaped particles starting from dimple particles as seeds. (b) Optical micrograph of the partially hollow dumbbellshaped particles in DMF in an AC electric field. Inset is the TEM image of the partially hollow dumbbell-shaped particles.

cavity, ${ }^{17,18,20}$ while the buckling mechanism relies on the presence of a uniform elastic shell. ${ }^{19,21}$ To study the distribution of cross-linked domains, PS-0.1h, PS-1.5h, and PS-6h were treated with dimethylformamide (DMF). DMF is capable of selectively dissolving linear polystyrene, while leaving cross-linked polystyrene unaffected. ${ }^{22}$ TEM images of the resulting etched colloids are shown in Figure $1 \mathrm{~g}-\mathrm{i}$. Based on the low contrast of the inner region of the colloids, we conclude that all particles became hollow, while a shell with a homogeneous thickness was preserved. The presence of this shell strongly indicates that shell buckling acts as the dominant driving force for dimple formation.

The generation of this core-shell structure can be rationalized by considering the elementary steps of dispersion polymerization and the particular experimental protocol that was followed. In a conventional dispersion polymerization, the monomer (St), stabilizer (PVP), and initiator are completely dissolved, yielding a homogeneous solution as the starting point. Upon initiation and polymerization, polymers are generated. After reaching a critical molecular weight, the polymers are no longer soluble in the continuous phase and hence precipitate to form nuclei. The initial nuclei are unstable and can be stabilized by absorbing the stabilizer to form stable particles. Subsequently, the colloids primarily grow by absorbing monomers from the continuous phase. Monomer absorption is driven by the fact that the hydrophobic monomer preferentially resides in the hydrophobic interior of the formed nuclei. Therefore, the main loci for polymerization are localized in the monomer-swollen particles (Figure 2d1). ${ }^{23}$ When DVB is added to the reaction mixture, the hydrophobic cross-linker molecules will also diffuse into the monomerswollen particles. However, since DVB is significantly more reactive than $\mathrm{St}^{24}$ the majority of DVB will be consumed before diffusing further into the particle's interior, yielding the observed core-shell morphology where a cross-linked shell engulfs a core composed of mainly linear polymers (Figure 2d2).

After formation of the core-shell structure, polymerization continues. Consumption of monomers in the monomerswollen core results in a volumetric contraction of the particle, which is larger for the linear core compared to the stiffer crosslinked shell. Since the core and shell are still attached to each other, this mismatch in contraction causes buckling of the shell and therefore cavity formation (Figure $2 \mathrm{~d} 3)^{21,25}$

According to the mechanism discussed above, we can explain the non-linear dependence of the cavity dimensions on the delayed addition time of DVB (Figure 2). If DVB is added at a too early stage ( $0.1 \mathrm{~h}$ after start of polymerization), the size of the primary particles is small, approximately one tenth of the final particle size. As a consequence, the particle radius $r$ over the shell thickness $s(r / s)$ is too small, prohibiting dimple formation since the volumetric contraction of the small core could hardly cause the buckling of the relatively thick shell. ${ }^{19}$ Additionally, the cross-linking density of the shell will be lower due to the presence of a high concentration of unreacted monomer. The mismatch in volumetric contraction between the shell and core during the polymerization will be limited, restricting the buckling process. When DVB is added toward the end of the polymerization ( $18 \mathrm{~h}$ after start of polymerization), the conversion and size of the colloids have nearly reached their maximum values upon addition of DVB as concluded from the kinetics data presented in Figure S1. Consequently, the monomer concentration in the core is low when the cross-linked shell is formed. Consuming this last fraction of monomer only yields a small volumetric contraction, which is not sufficient to form a cavity. Therefore, smooth and spherical monodisperse microspheres were obtained. In between 0.1 and $18 \mathrm{~h}$, with the time increasing, $r / s$ increases, which promotes the buckling process. ${ }^{9}$ However, the magnitude of volumetric contraction caused by monomer consumption decreases with time, prohibiting buckling. The interplay between these two competing contributions results in the behavior plotted in Figure 2, where the maximum cavity size was obtained after an addition time of $1.5 \mathrm{~h}$. Since $r / s$ is the dominating factor in determining if buckling will occur and this parameter is readily tunable by the delay time after which the monomer was added (Figure 2c), direct control over the cavity dimensions is possible.

To strengthen our claims on the volumetric contraction of the core to be a key parameter in the buckling of the crosslinked shell, the cavity evolution as a function of time was monitored. Figure 3 shows the $L / r$ value of PS- $1.5 \mathrm{~h}$ as a function of time where $L$ is defined as the diameter of the cavity and $r$ as the radius of the colloidal particle (Figure $2 \mathrm{~d}$ ). Cavities became observable $1.5 \mathrm{~h}$ after addition of cross-linkers (Figure 3c). Furthermore, the dimple particles obtained after $24 \mathrm{~h}$ of polymerization show cavities that are significantly larger than those obtained after $3.25 \mathrm{~h}$ of polymerization (Figure 3a,b). This cavity growth is in agreement with the continuous contraction of the core due to the gradual consumption of monomers in the core.

3.3. Influence of Cross-Linking Density. Next, we investigated the influence of cross-linking density of the shell on the resulting particle morphology. Based on the previously presented evidence that cavity formation is caused by shell buckling, we anticipated a distinct influence of this experimental parameter on the dimple dimensions. To this 
end, particles with a molar ratio of total styrene to DVB ranging from 210:1 to 5:1 were prepared. For this set of experiments, the addition time of DVB was fixed at $1.5 \mathrm{~h}$ after the start of the initial polymerization. When the molar ratio of total styrene to DVB is high (210:1), hemispherical colloids with no or very shallow cavities were obtained (Figure 4a). When this ratio is lowered to a range roughly in between 100:1 and 25:1, particles with one well-defined cavity were formed (Figure 4b,c). The average diameter of the cavity decreases from 500 to $400 \mathrm{~nm}$ within this cross-linker concentration window. Decreasing the molar ratio of styrene to DVB even further down to 10:1 and 5:1 gave spherical colloids with no cavity (Figure 4d). From this set of data, we can conclude that there exists an experimental window of cross-linker concentrations in which cavity formation is favored (molar ratio of total styrene to DVB between approximately 100:1 and 25:1, Figure 4). Furthermore, the findings presented in Figure 4 are consistent with the buckling mechanism. The lack of any cavity at low cross-linking densities is due to the shell's inability to generate sufficient elastic stress to buckle the particle. If the cross-linking density becomes too high, buckling is hampered by the rigidity of the shell. Interestingly, in this case, hollow spherical particles are obtained, further demonstrating that the core contracts during polymerization (Figure $4 \mathrm{~d}$ and Figure S2).

3.4. Synthesis of Dimple Particles with Various Surface Functionalities. Since it was established that dimple formation solely relies on the buckling of an elastic cross-linked outer shell, we anticipated that our approach could be extended to access chemically functionalized dimple particles. The introduction of chemical handles for further surface modifications will greatly extend the application scope of dimple particles. ${ }^{13,13}$ To introduce chemical surface functionalities, additional (functional) comonomers are added to the polymerization mixture together with DVB during the delayed addition process. This strategy was selected to maximize the surface exposure of the target chemical functionalities. To illustrate the robustness of this approach, dimple particles with benzyl chloride moieties (PS-PVBC) and epoxide groups (PSPGMA) were synthesized by using vinylbenzyl chloride (VBC) or glycidyl methacrylate (GMA) as comonomers, respectively. The benzyl chloride and epoxide moieties are versatile chemical handles and give access to further surface modifications via nucleophilic substitutions and ring opening reactions, respectively. Besides these styrene and methacrylatebased monomers, $N$-isopropylacrylamide (NIPAM) was also selected as a functional comonomer, providing access to thermo-responsive dimple colloids.

Regardless of the chemical nature of the comonomers, welldefined dimple particles were obtained, clearly indicating that the presence of these comonomers has no measurable influence on dimple formation (Figure 5). The incorporation of functional groups in the dimple particles was confirmed by infrared (IR) spectroscopy (Figure 5d). Compared to the IR spectrum of non-functionalized dimple particles, the spectra of PS-PVBC, PS-PGMA, and PS-PNIPAM revealed diagnostic vibrational bands at $1266 \mathrm{~cm}^{-1}\left(-\mathrm{CH}_{2}-\mathrm{Cl}\right), 1730 \mathrm{~cm}^{-1}(\mathrm{C}=$ $\mathrm{O}$ ester stretch), and $1676 \mathrm{~cm}^{-1}(\mathrm{C}=\mathrm{O}$ amide stretch), respectively. $^{26}$

To further confirm that the functional groups are indeed concentrated on the surface and are accessible to subsequent chemical modifications, PS-PVBC was treated with fluoresceinamine. This dye carries a primary amine group that is capable of reacting with the benzyl chloride functionalities. The resulting dye-labeled particles were imaged by confocal microscopy. The green signal originating from the fluorescence of the attached dye is evenly distributed over the particle surface, clearly showing the dimpled contour of the particles and suggesting a uniform distribution of the pendant benzyl chloride functionalities over the particle surface (Figure 5e).

3.5. Synthesis and Assembly of Partially Hollow Dumbbell-Shaped Particles. In this final section, the unique core-shell structure of the prepared dimple particles will be leveraged to synthesize a new class of model colloids for (self)assembly, namely, particles that are anisotropic in their density. Following a previously reported procedure, ${ }^{10}$ the dimple seeds were swollen with a mixture of St and DVB and subsequently heated to induce phase separation between the cross-linked shells of the seeds and the swelling monomer to yield particles with a liquid protrusion. These protrusions are then polymerized to yield dumbbell-shaped colloids. In the final step, these dumbbells were etched with DMF to yield particles with one hollow lobe derived from the dimple seed (tail) and one solid lobe originating from the homogeneously cross-linked protrusion (head), as depicted in Figure 6.

In an external AC electric field, colloids acquire a dipole moment that is oriented parallel to the applied electric field as a result of the dielectric contrast between the colloids and the solvent. $^{27,28}$ The magnitude of the induced dipole moment is proportional to the particle volume, leading to stronger dipole moments for larger particles. ${ }^{28}$ If the induced dipoles are sufficiently large, dipole-dipole interactions will force the particle to align. In the case of the partially hollow dumbbellshaped particles, the induced dipoles are expected to be asymmetric. For the solid lobe a stonger dipole was anticipated, as more polymeric material contributes to the dielectric contrast compared to the solvent-filled hollow lobes. Consequently, dipole-dipole interactions between two solid lobes are stronger than the interactions acting between two hollow lobes or a combination of a solid and a hollow lobe. To verify the directionality in the dipole-dipole induced interactions, the assembly behavior of the obtained partially hollow dumbbell-shaped particles dispersed in DMF was studied by applying an external electric field $(40 \mathrm{~V} / \mathrm{mm}$, frequency of $1 \mathrm{MHz}$ ). DMF was selected as dispersing medium, as this resulted in a significant optical contrast between the smaller, filled heads and larger hollow tails (Figure $6 \mathrm{~b})$. This contrast difference is advantageous in tracking the relative orientation between the colloids after alignment. As depicted in Figure 6b, in an AC electric field, as expected, most particle chains have the heads in closest contact. Overall, approximately $80 \%$ of the hollow dumbbell-shaped particles are aligned perpendicular to the electric field. Furthermore, as shown before, the dimple particles can be functionalized by reactive functional groups. This allows the synthesis of chemically anisotropic partially hollow dumbbell-shaped particles and therefore opens avenues to fixate the formed chains to generate new supra-colloidal objects (see Supporting Information S3 for the details).

\section{CONCLUSIONS}

Monodisperse, micron-sized dimple particles with a single welldefined cavity are synthesized by delayed addition of crosslinkers into a dispersion polymerization system. The mechanism of the dimple formation is elucidated as follows: First, by delayed addition of cross-linkers, colloids with a 
distinct core-shell structure, which consists of a core rich in linear polymers surrounded by a cross-linked outer shell, are obtained. This core-shell structure is confirmed by selective DMF-mediated etching of the non-cross-linked polymers. Subsequently, the elastic shell buckles during the ongoing polymerization as the monomer swollen core contracts upon consumption of the monomer, driving the formation of dimples via a buckling process. The validity of this mechanism is confirmed by showing that the cavity size is readily tunable by variation of the delayed addition time of cross-linkers as well as its overall concentration. Furthermore, we show that this method can be extended in a straightforward way to synthesize dimple particles with various surface functionalities by introducing additional functional comonomers together with cross-linkers during the delayed addition step. Lastly, the unique core-shell dimple colloids are used as seeds for the preparation of (chemically) anisotropic partially hollow dumbbell-shaped colloids with one hollow and one solid lobe, which are promising building blocks for directed (self)assembly in an externally applied AC electric field.

\section{ASSOCIATED CONTENT}

\section{S Supporting Information}

The Supporting Information is available free of charge on the ACS Publications website at DOI: 10.1021/acs.macromol.9b00406.

Time evolution of the particle size and monomer conversion of a dispersion polymerization of solely styrene, TEM image of particles synthesized using a molar ratio of total styrene to DVB of $10: 1$, and synthesis of chemically anisotropic partially hollow dumbbell-shaped particles (PDF)

\section{AUTHOR INFORMATION}

\section{Corresponding Author}

*E-mail: w.k.kegel@uu.nl.

\section{ORCID}

Yong Guo: 0000-0002-5609-5550

Bas G. P. van Ravensteijn: 0000-0001-9024-3927

\section{Present Address}

${ }^{\dagger}$ Netherlands Organisation for Applied Scientific Research (TNO), Materials Solutions, High Tech Campus 25, NL 5600 HE Eindhoven, The Netherlands

\section{Notes}

The authors declare no competing financial interest.

\section{ACKNOWLEDGMENTS}

Pepijn Moerman is acknowledged for operating the electric field experiment and for useful discussion. Sonja Castillo is acknowledged for taking the scanning electron microscopy images. Y.G. is supported by a scholarship under the State Scholarship Fund (file no. 201306200056) from the Chinese government.

\section{REFERENCES}

(1) Lu, Y.; Yin, Y.; Xia, Y. Three-dimensional photonic crystals with non-spherical colloids as building blocks. Adv. Mater. 2001, 13, 415420.

(2) Glotzer, S. C.; Solomon, M. J. Anisotropy of building blocks and their assembly into complex structures. Nat. Mater. 2007, 6, 557-562.

(3) Chen, Q.; Bae, S. C.; Granick, S. Directed self-assembly of a colloidal kagome lattice. Nature 2011, 469, 381-384.
(4) Duguet, E.; Désert, A.; Perro, A.; Ravaine, S. Design and elaboration of colloidal molecules: an overview. Chem. Soc. Rev. 2011, 40, 941-960.

(5) Fan, J.-B.; Song, Y.; Li, H.; Jia, J.-P.; Guo, X.; Jiang, L. Controllable drug release and effective intracellular accumulation highlighted by anisotropic biodegradable PLGE nanoparticles. J. Mater. Chem. B 2014, 2, 3911-3914.

(6) Paxton, W. F.; Kistler, K. C.; Olmeda, C. C.; Sen, A.; St. Angelo, S. K.; Cao, Y.; Mallouk, T. E.; Lammert, P. E.; Crespi, V. H. Catalytic Nanomotors: Autonomous Movement of Striped Nanorods. J. Am. Chem. Soc. 2004, 126, 13424-13431.

(7) Wensink, H. H.; Kantsler, V.; Goldstein, R. E.; Dunkel, J. Controlling active self-assembly through broken particle-shape symmetry. Phys. Rev. E 2014, 89, No. 010302.

(8) van Ravensteijn, B. G. P.; Kamp, M.; van Blaaderen, A.; Kegel, W. K. General Route toward Chemically Anisotropic Colloids. Chem. Mater. 2013, 25, 4348-4353.

(9) Kim, J.-W.; Larsen, R. J.; Weitz, D. A. Synthesis of Nonspherical Colloidal Particles with Anisotropic Properties. J. Am. Chem. Soc. 2006, 128, 14374-14377.

(10) Wolters, J. R.; Avvisati, G.; Hagemans, F.; Vissers, T.; Kraft, D. J.; Dijkstra, M.; Kegel, W. K. Self-assembly of "Mickey Mouse" shaped colloids into tube-like structures: experiments and simulations. Soft Matter 2015, 11, 1067-1077.

(11) Rossi, L.; Sacanna, S.; Irvine, W. T. M.; Chaikin, P. M.; Pine, D. J.; Philipse, A. P. Cubic crystals from cubic colloids. Soft Matter 2011, 7, 4139-4142.

(12) Zhang, Z.; Pfleiderer, P.; Schofield, A. B.; Clasen, C.; Vermant, J. Synthesis and Directed Self-Assembly of Patterned Anisometric Polymeric Particles. J. Am. Chem. Soc. 2011, 133, 392-395.

(13) She, S.; Li, Q.; Shan, B.; Tong, W.; Gao, C. Fabrication of RedBlood-Cell-Like Polyelectrolyte Microcapsules and Their Deformation and Recovery Behavior Through a Microcapillary. Adv. Mater. 2013, 25, 5814-5818.

(14) Sacanna, S.; Irvine, W. T. M.; Chaikin, P. M.; Pine, D. J. Lock and key colloids. Nature 2010, 464, 575-578.

(15) Doshi, N.; Zahr, A. S.; Bhaskar, S.; Lahann, J.; Mitragotri, S. Red blood cell-mimicking synthetic biomaterial particles. Proc. Natl. Acad. Sci. 2009, 106, 21495-21499.

(16) Sacanna, S.; Irvine, W. T. M.; Rossi, L.; Pine, D. J. Lock and key colloids through polymerization-induced buckling of monodisperse silicon oil droplets. Soft Matter 2011, 7, 1631-1634.

(17) Huang, Y.; Wang, J.; Zhou, J.; Xu, L.; Li, Z.; Zhang, Y.; Wang, J.; Song, Y.; Jiang, L. Controllable Synthesis of Latex Particles with Multicavity Structures. Macromolecules 2011, 44, 2404-2409.

(18) Cheng, Z.; Luo, F.; Zhang, Z.; Ma, Y.-q. Syntheses and applications of concave and convex colloids with precisely controlled shapes. Soft Matter 2013, 9, 11392-11397.

(19) Peng, B.; Imhof, A. Surface morphology control of cross-linked polymer particles via dispersion polymerization. Soft Matter 2015, 11, $3589-3598$.

(20) Xie, D.; Ren, X.; Xie, Y.; Zhang, X.; Liao, S. Large-Scale Synthesis of Monodisperse Red Blood Cell (RBC)-Like Polymer Particles. ACS Macro Lett. 2016, 5, 174-176.

(21) Meester, V.; Kraft, D. J. Spherical, Dimpled, and Crumpled Hybrid Colloids with Tunable Surface Morphology. Langmuir 2016, 32, 10668-10677.

(22) van Ravensteijn, B. G. P.; Kegel, W. K. Tuning particle geometry of chemically anisotropic dumbbell-shaped colloids. $J$. Colloid Interface Sci. 2017, 490, 462-477.

(23) Tseng, C. M.; Lu, Y. Y.; El-Aasser, M. S.; Vanderhoff, J. W. Uniform polymer particles by dispersion polymerization in alcohol. $J$. Polym. Sci., Part A: Polym. Chem. 1986, 24, 2995-3007.

(24) Hild, G.; Rempp, P. Mechanism of network formation by radical copolymerization. Pure Appl. Chem. 1981, 53, 1541.

(25) Zoldesi, C. I.; Imhof, A. Synthesis of Monodisperse Colloidal Spheres, Capsules, and Microballoons by Emulsion Templating. Adv. Mater. 2005, 17, 924-928. 
(26) Socrates, G. Infrared and Raman Characteristic Group Frequencies, 3rd Edition.; John Wiley \& Sons Ltd, 2001.

(27) van Blaaderen, A.; Dijkstra, M.; van Roij, R.; Imhof, A.; Kamp, M.; Kwaadgras, B. W.; Vissers, T.; Liu, B. Manipulating the self assembly of colloids in electric fields. Eur. Phys. J. Spec. Top. 2013, 222, 2895-2909.

(28) Smallenburg, F.; Vutukuri, H. R.; Imhof, A.; van Blaaderen, A.; Dijkstra, M. Self-assembly of colloidal particles into strings in a homogeneous external electric or magnetic field. J. Phys.: Condens. Matter 2012, 24, 464113. 